\title{
Combined Antenna and Localized Plasmon Resonance in Raman Scattering from Random Arrays of Silver-Coated, Vertically Aligned Multiwalled Carbon Nanotubes
}

\author{
P. Dawson, ${ }^{*}$ J. A. Duenas, ${ }^{\dagger}$ M. G. Boyle, M. D. Doherty, and S. E. J. Bell \\ Centre for Nanostructured Media, School of Maths and Physics, Queen's University of Belfast, Belfast BT7 1NN, U.K.
}

\author{
A. M. Kern and O. J. F. Martin \\ Nanophotonics and Metrology Laboratory, Ecole Polytechnique de Lausanne, CH-1015 Lausanne, Switzerland
}

\begin{abstract}
A.-S. Teh, K. B. K. Teo, and W. I. Milne
Centre for Applied Photonics and Electronics, Cambridge University, $9 \mathrm{JJ}$ Thomson Avenue, Cambridge CB3 OFA
\end{abstract}

Supporting Information

\begin{abstract}
The electric field enhancement associated with detailed structure within novel optical antenna nanostructures is modeled using the surface integral equation technique in the context of surface-enhanced Raman scattering (SERS). The antennae comprise random arrays of vertically aligned, multiwalled carbon nanotubes dressed with highly granular Ag. Different types of "hot-spot" underpinning the SERS are identified, but contrasting characteristics are revealed. Those at the outer edges of the Ag grains are antenna driven with field enhancement amplified in antenna antinodes while intergrain hotspots are largely independent of antenna activity. Hot-spots between the tops of antennae leaning towards each other also appear to benefit from antenna amplification.
\end{abstract}
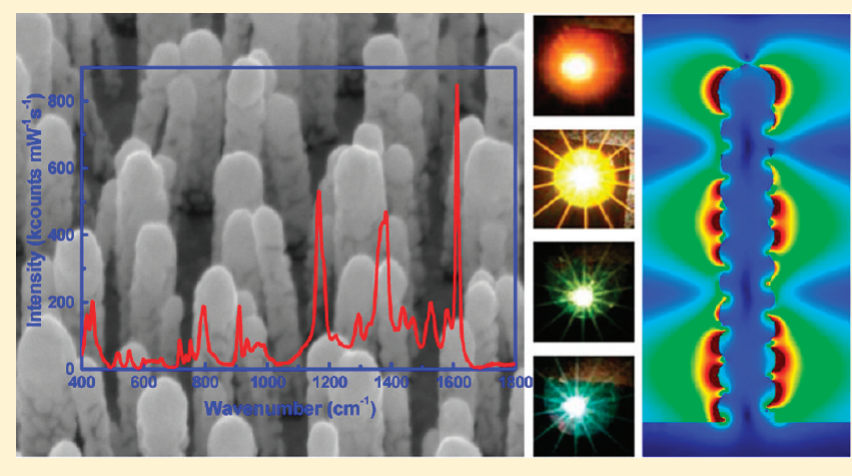

KEYWORDS: Surface-enhanced Raman scattering, optical antenna, plasmons, surface integral equation, carbon nanotubes

$\mathrm{T}$ he phenomenon of surface-enhanced Raman scattering (SERS) was first observed in $1974,{ }^{1}$ while the discovery of the multiwalled carbon nanotube (MWNT) followed some 17 years later in $1991 .^{2}$ Substrates used in SERS have been the subject of intensive scrutiny in terms of fabrication and analysis with emphasis being focused on the electromagnetic field enhancement due to localized plasmon resonances associated with the highly profiled metallic substrates. ${ }^{3,4}$ Such substrates have ranged from highly roughened metal films or electrodes ${ }^{1,5-7}$ to metallic structures formed using techniques based on self-assembly, notably nanosphere lithography, ${ }^{8,9}$ and those based on standard photolithography and electron beam lithography where, for example, very precisely defined metallic islands or antenna have been formed. ${ }^{10-12}$ In recent years silver and gold nanowires grown in porous alumina substrates have attracted interest as potential SERS substrates on account their plasmon-active nature. ${ }^{13-18}$

In the context of carbon nanotube applications, very significant effort has been directed toward nanotube functionalization. ${ }^{19}$ This can involve a wide range of treatments, typically with a view to the attachment of a species of interest ${ }^{20}$ or to modification of the band structure (of single-walled nanotubes) for electronic device applications. ${ }^{21}$ In this investigation, random arrays of vertically aligned, MWNTs are used as a framework for a thin Ag overlayer to act as a SERS substrate. This silver-coating treatment may be regarded as a simple, convenient, and cheap means of functionalizing MWNTs for SERS.

The growth of the MWNTs follows a "bottom-up" methodology but with contrasting degrees of self-assembly in the two stages of the process. Ni nanoparticles that act as the catalytic growth centers for the MWNTs were formed by thermally annealing a thin film of $\mathrm{Ni}$ deposited on a silicon substrate while the MWNT growth itself was effected by means of plasmaenhanced chemical vapor deposition. ${ }^{22,23}$ The MWNT substrates were then mounted in a thermal evaporator and coated with

Received: August 11, 2010

Revised: November 25, 2010

Published: January 25, 2011 

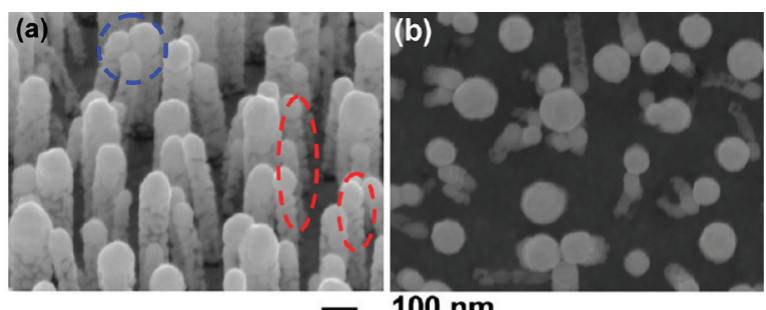

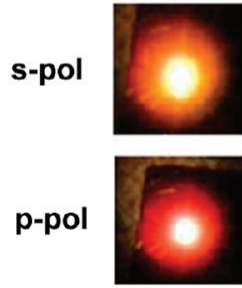

$20^{\circ}$

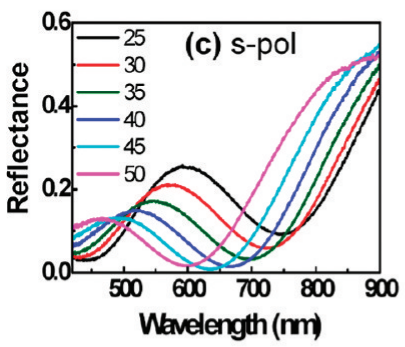

$30^{\circ}$
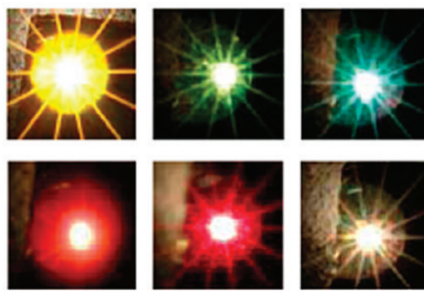

$40^{\circ}$

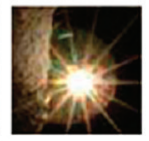

$50^{\circ}$
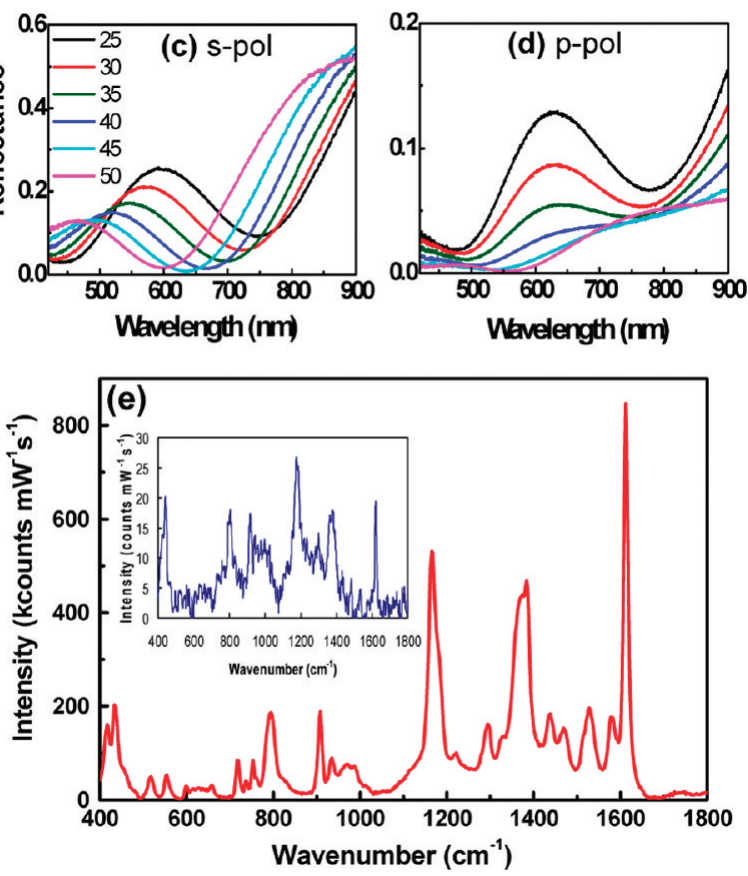

Figure 1. SEM, optical, and Raman data for a sample comprising a random array of vertically aligned MWNTs coated with Ag. SEM images taken at (a) $45^{\circ}$ and (b) normal incidence. Features enclosed by red and blue dashed lines in (a) are discussed in the text. Photographic insert: specular reflection from sample illuminated by beam of collimated, $\mathrm{s}-(\mathrm{p}-)$ polarized white light at angles of incidence indicated, captured on standard digital camera. Reflectance spectra for (c) s-polarized and (d) p-polarized light at angles of incidence indicated in the key in (c). (e) Raman spectrum from crystal violet (CV) deposited on Ag-surface of sample taken with Raman microscope using input laser of wavelength $632.8 \mathrm{~nm}$ and power $0.02 \mathrm{~mW}$. Spectral intensity has been normalized to k-counts $\mathrm{mW}^{-1} \mathrm{~s}^{-1}$. (Inset in e) Calibration spectrum recorded from drop of CV of $10^{-3} \mathrm{M}$ concentration deposited on $\mathrm{Ag}$ thin film.

Ag at a rate of $\sim 0.5 \mathrm{~nm} \mathrm{~s}^{-1}$ by rotation at a frequency of $\sim 1 \mathrm{~Hz}$ with the substrate normal at an angle of $\sim 15^{\circ}$ to the direction of the arriving evaporant. For the substrate investigated in detail here, the average length of the Ag-coated MWNTs (or nanoposts) is $L_{\text {SEM }}=510 \mathrm{~nm}$ and the average diameter is $D_{\text {SEM }}=80 \mathrm{~nm}$, as assessed from SEM images (Figure 1a,b); the uncoated MWNTs had an average diameter of $\sim 57 \mathrm{~nm}$. The packing density is relatively low with a fill-factor on the underlying silicon surface being just under 0.2 (Figure $1 \mathrm{~b}$ ). A further feature of critical importance is that the Ag coating of the nanoposts is highly granular in nature. Given this structure, the purpose of the present study is to explore by a combination of experiment and modeling, how localized plasmon resonances associated with the granular Ag coating combine with the overall nanopost antenna response to contribute to the SERS enhancement factor (EF).

Crystal violet (CV) was used as the test molecule in the Raman spectroscopy. The molecules were deposited by dropping $1 \mu \mathrm{L}$ of $5 \times 10^{-5} \mathrm{M}$ solution on the sample surface which was then allowed to dry. Various concentrations of $\mathrm{CV}$ solution were tested with the selected concentration being just above that at which a plot of Raman signal versus concentration reached its maximum (saturation) level; we comment further on this below. Raman spectra were recorded with a Jobin-Yvon LabRam Raman microscope using a $50 \times$ objective of numerical aperture 0.55 with input laser light of wavelength, $\lambda=632.8 \mathrm{~nm}$; the laser power incident on the sample surface was typically in the range $0.02-0.2 \mathrm{~mW}$.

The core experimental results are summarized in Figure 1, comprising the SEM images (Figure 1a,b), as already discussed above, the elastic optical response (color insert and Figure 1c,d) and Raman spectra (Figure 1e). Before proceeding to the detailed modeling of the field enhancement underpinning the SERS EF, it is needful to address some basic optical properties that are input to the modeling and the evaluation of the EF itself. There are four points involved here, all with more detailed development in the Supporting Information.

(1) First, the nanopost substrates exhibit striking coloration effects as a function of angle of incidence and polarization. These are illustrated in the color inset and characterized quantitatively in terms of the specular reflectance in Figure $1 \mathrm{c}, \mathrm{d}$. While the system could be analyzed as an array of "core-shell" entities ${ }^{24,25}$ of cylindrical geometry, a simple model is developed in the Supporting Information where the nanopost "layer" is treated as an effective medium. The s-polarized reflectance spectra are well reproduced, conforming to a thin film interference effect with scattering losses. The principal outcome is that there is a slight upward adjustment of the nanopost length and diameter to $L=525 \mathrm{~nm}$ and $D=85 \mathrm{~nm}$ (subscripts dropped) from the values estimated from the SEM images. Points (2) and (3) address two further important optical factors to render the modeling more realistic; these pertain to the outer and inner environments of the $\mathrm{Ag}$ nanopost.

(2) The substrate is "overdosed" with CV with perhaps a coating of thickness up to $\sim 10 \mathrm{~nm}$ present on the surface. This is suggested on two main points of evidence, one in relation to known surface molecular coverage ${ }^{26}$ and the other in relation to the experimental conditions. These points and the use of a refractive index, $n_{\mathrm{CV}}=1.50$ are discussed in the Supporting Information.

(3) The modeling develops from treating the nanoposts as being composed purely of $\mathrm{Ag}$ to the insertion of the MWNT core. From a brief consideration of the optical properties of various forms of carbon ${ }^{27-32}$ (see Supporting Information) we opt to describe the MWNT with the refractive index given by Bruna and Borini; ${ }^{31}$ this has the form $n_{\mathrm{MWNT}}=3.0+i(\mathrm{C} / 3.0) \lambda$, which with $C=$ $5.445 \mu \mathrm{m}^{-1}$ yields a value of $n_{\mathrm{MWNT}}=3.0+i 1.15$ at $\lambda=$ $632.8 \mathrm{~nm}$. 
(4) Raman spectra of CV acquired from the nanopost substrates (Figure 1e) exhibit three strong lines at 1165, 1383 , and $1612 \mathrm{~cm}^{-1}$ with peak intensities in the range $(6.5 \pm 2.0) \times 10^{5}$ counts $\mathrm{mW}^{-1} \mathrm{~s}^{-1}$, corresponding to signal enhancements, relative to the reference spectrum (Figure 1e, inset), of $(1.3 \pm 0.5) \times 10^{5}$. Realistic, accurate evaluation of the absolute EF in SERS is a pivotal issue $^{9,33,34}$ and critically relevant to the modeling that follows; this is addressed in the Supporting Information where it is shown that the EF lies in the range $(5.2 \pm 2.0) \times$ $10^{3}$ with reference to the case of a droplet of $10^{-3} \mathrm{M}$ solution of $\mathrm{CV}$ on a flat $\mathrm{Ag}$ substrate. This value takes into account an areal enhancement of $5.0 \pm 0.2$ of the structured substrate relative to the planar Ag substrate. However, the source of the SERS signal is not evenly distributed throughout the estimated $10 \mathrm{~nm}$ CV coverage of point (2). From dosing experiments at the outset, it is known that most $(\sim 70 \%)$ of the SERS signal can be retrieved with surface coverage that is almost $10 \times$ less than that used in Figure 1. In effect, the SERS EF at the surface (for the first monolayer) could be $7 \times$ higher at $(3.6 \pm 1.4) \times 10^{4}$, requiring an average uniform field enhancement in the range $12-15$. However, in stating this it should be noted that the SERS EF is given (approximately) by $\left\langle\left|E_{\mathrm{Loc}}\right|^{4}\right\rangle$, which is significantly greater than $\left\langle\left|E_{\mathrm{Loc}}\right|\right\rangle^{4}$, where $\left|E_{\mathrm{Loc}}\right|$ denotes the magnitude of the local electric field and the angled brackets denote the averaged surface value. We use an $\left|E_{\mathrm{Loc}}\right|^{4}$ dependence (evaluated at the stimulation wavelength) as a reasonable approximation in order to save significant computation time that would be incurred by calculation of $\left|E_{\mathrm{Loc}}\right|^{2}$ at both incident and scattered wavelengths. The remainder of this article focuses on the modeling of the electric field enhancement for realistic nanopost structures with the aim of explaining the SERS $\mathrm{EF}$ and understanding the salient details of its origin.

Such modeling of the electromagnetic response should take into account the granular nature of the $\mathrm{Ag}$ nanopost surface. Thus, a 3D surface integral equation (SIE) technique was used. On the basis of the PMCHWT (Poggio, Miller, Chang, Harrington, $\mathrm{Wu}$, and Tsai) formulation ${ }^{35}$ adapted to consider composite objects, $^{36}$ this routine is well suited to simulate plasmonic and resonant phenomena. Surface meshing allows restriction of the grid refinement to where needed, facilitating the simulation of complex geometric structures with reasonable computational costs. As the simulation domain of integral equation techniques extends to infinity, simulated objects are modeled as having a finite size. In the present case, the nanopost and substrate are treated as a single finite entity, but the substrate portion must be large enough that its finite nature has no effect on the near-field of the nanopost, that is, relative to the nanopost on a semi-infinite substrate.

Thus, before addressing the nanopost system of Figure 1 using the SIE technique, it is necessary to consider the effect of (a) a finite substrate and (b) interaction with neighboring nanoposts. This is done by assuming smooth post structures formed from pure $\mathrm{Ag}$ and performing the modeling of the electric field intensity using a commercial finite element modeling package, COMSOL 3.5a. The optical response of pure Ag nanoposts will give a reasonable first approximation to that of nanoposts comprising MWNTs with an average Ag coating of thickness $\sim 10 \mathrm{~nm}$ (or almost half a skin-depth); the MWNT core is inserted at a later stage and the effect of its perturbation assessed. Figure 2

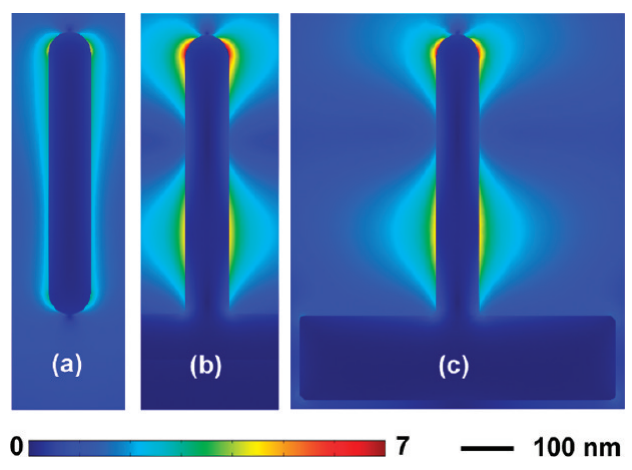

Figure 2. Plots of electric field intensity, calculated using finite element method, due to illumination of model systems comprising smooth $\mathrm{Ag}$ posts with $L=525 \mathrm{~nm}$ and $D=82 \mathrm{~nm}$. Light of wavelength $632.8 \mathrm{~nm}$ is incident at $0^{\circ}$, that is, along the direction of the nanopost long axis with electric field polarized in the plane of incidence, which is also that of the cross sections: (a) isolated $\mathrm{Ag}$ nanopost with hemispherical ends, (b) Ag nanopost on semi-infinite $\mathrm{Ag}$ substrate, and (c) $\mathrm{Ag}$ nanopost on finite $\mathrm{Ag}$ disk of diameter $600 \mathrm{~nm}$ and thickness $150 \mathrm{~nm}$.

illustrates the electric field intensity for a smooth $\mathrm{Ag}$ post with $L=$ $525 \mathrm{~nm}, D=82 \mathrm{~nm}$ (i.e., reduced slightly from $85 \mathrm{~nm}$ to reflect the average or smoothed post profile); plane-polarized light $(\lambda=$ $632.8 \mathrm{~nm}$ ) is incident at $\theta_{i}=0^{\circ}$ (i.e., in the direction of the nanopost long axis) where the plane of polarization is the same as that of the cross-sectional view. The image of Figure $2 \mathrm{a}$ is for the case of an isolated post (with hemispherical ends) in free space, while Figure $2 \mathrm{~b}$ illustrates the case where the same nanopost is connected to a semi-infinite Ag substrate, taken to represent the experiment since $\mathrm{Ag}$ is deposited to somewhat greater thickness on the silicon substrate than on the sidewalls of the MWNTs. Both the intensity and distribution of the electric field are substantially modified by the semi-infinite Ag base; in particular, a distinct maximum (4-5 times the incident field) appears about one-third the way up the nanopost, as well as a region of higher field intensity at the top of the structure. The next step in the preliminary modeling is to introduce a Ag disk of finite size that adequately replicates the effect of a semi-infinite base, but that can be accommodated within the SIE technique without incurring excessive computation time. This situation is achieved in Figure $2 \mathrm{c}$ with a substrate disk of diameter $600 \mathrm{~nm}$ and thickness $150 \mathrm{~nm}$; this defines the finite substrate that is used in all subsequent SIE calculations.

Figure 3 addresses the issue of interaction between the nanoposts. Figure 3 a comprises a set of images that illustrate the cases where plane-polarized light is incident on an isolated Ag nanopost (on a semi-infinite $\mathrm{Ag}$ substrate) at angles of incidence $\theta_{i}=0^{\circ}$ (left image) and at $\theta_{i}=25^{\circ}$ for light that is s-polarized (central image) or p-polarized (right image). The two angles chosen in Figure 3 (and hereafter) are intended to be representative of the lower and higher angle input range from the objective in the Raman microscope; with a numerical aperture of 0.55 the half angle of the incident light cone focused on the sample is $33.4^{\circ}$. Figure $3 \mathrm{~b}$ illustrates the equivalent cases to Figure $3 \mathrm{a}$, except that the nanopost is now in an array of period $160 \mathrm{~nm}$, corresponding to the average observed spacing between the nanoposts. Thus, although the array is periodic, (rather than random), there is a large mismatch between the component of the light wave vector parallel to the surface and any (low-order) periodicity vector associated with the nanopost array, avoiding any obvious photonic crystal effects. From Figure $3 b$, it is clear that the internanopost 
(a) $0^{\circ}$

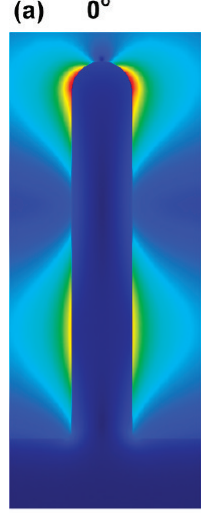

(b) $0^{\circ}$

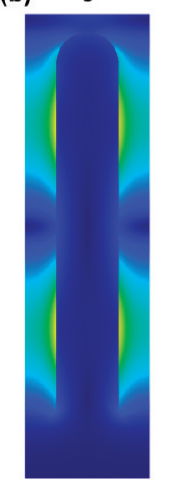

0 $25^{\circ}$ s-pol

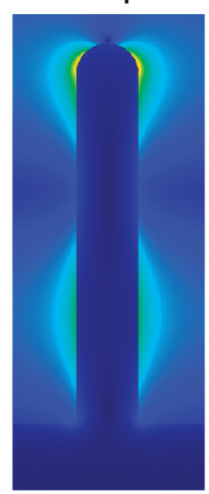

$25^{\circ}$ s-pol

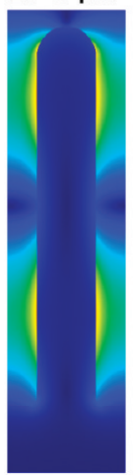

$25^{\circ} \mathrm{p}-\mathrm{pol}$

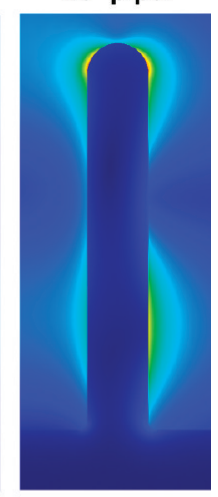

$25^{\circ} \mathrm{p}$-pol

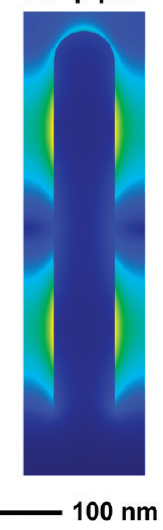

Figure 3. Plots of electric field intensity, calculated using finite element method, due to illumination of model systems comprising smooth $\mathrm{Ag}$ posts with $L=525 \mathrm{~nm}$ and $D=82 \mathrm{~nm}$ on semi-infinite $\mathrm{Ag}$ substrate. (a) Case of isolated posts under illumination with light of wavelength 632.8 $\mathrm{nm}$ incident at $0^{\circ}$ (left image) and at $25^{\circ}$ (s-polarization, middle image; p-polarization, right image); light is incident from upper left side in $25^{\circ}$ p-polarization case. Cross sections are in the same plane as the polarization vector. (b) As for part (a) but with $\mathrm{Ag}$ nanopost now located in an array of periodicity $160 \mathrm{~nm}$.

interaction "pulls" the electric field into a much more symmetrical distribution than that pertaining to the isolated posts, especially for the p-polarized case at $\theta_{i}=25^{\circ}$. While the presence of neighboring nanoposts reduces the peak field intensity at $\theta_{i}=0^{\circ}$, it increases the field enhancement at $\theta_{i}=25^{\circ}$. The lack of interpost interactions is the main approximation in considering the modeling of single, lumpy nanopost structures below.

The next significant step in the modeling is to accommodate the granular nature of the Ag coating using the SIE method. The $\mathrm{Ag}$ layer is treated as a collection of overlapping spherical particles where the overlap is such that the MWNT is just covered with Ag at all points. The spherical sections are of radius $25 \mathrm{~nm}$ with the sphere centers arranged in a helix of radius $17.5 \mathrm{~nm}$ and pitch $45 \mathrm{~nm}$. The helix construct lies within a MWNT core, such that the final structure comprises a $60 \mathrm{~nm}$ diameter MWNT core coated with spherical sections of $\mathrm{Ag}$ of maximum thickness of $12.5 \mathrm{~nm}$, giving a nanopost diameter of $85 \mathrm{~nm}$ to the extremities of the $\mathrm{Ag}$. This structure has approximately the correct nominal thickness of $\mathrm{Ag}$ coating and displays $\sim 11 \mathrm{Ag}$ particles along the length of sectional views, which is in agreement with the SEM images; a hemispherical Ag cap is retained on the tops of the posts.

First, it is instructive to continue to treat the entire nanopost structure as composed purely of Ag. The resulting field intensity plots of Figure 4a, in comparison with those of Figure 3a for smooth Ag nanoposts, demonstrate that regions of enhanced field associated with the Ag grains develop within the modulating envelope of the overall antenna field profile. This is particularly evident in the lower antinode where the peak enhancement occurs at the protruding outer edges of the Ag grains and exceeds a factor of 8 , compared to $\sim 4-5$ in the case of equivalent smooth post structures. Importantly, we note also that for an array of spherical particles similarly protruding from a planar Ag surface (the "unrolled" antenna case), the maximum field enhancement is again in the range of $4-5$ (not shown). Thus, it is clear that in the antenna format of Figure 1a the Ag particles are fed from the antenna excitation with field maxima in the antinodal regions markedly exceeding that of a planar array of such particles or that of the smooth antenna structure of Figure 3, while there is virtually no field enhancement in the nodal regions. This antennadriven redistribution of field enhancement (relative to the case of Ag protrusions on a planar substrate) is highly advantageous for SERS on account of the $\left|E_{\mathrm{Loc}}\right|^{4}$ dependence of the EF.

Developing point (2) above in conjunction with the fact that the greatest field enhancement lies within $\sim 10 \mathrm{~nm}$ of the surface the next step is to approximate the ambient medium to $n_{\mathrm{CV}}=1.5$. This alters the distribution of field enhancement as shown in Figure $4 b$; the main features are a change in the effective wavelength of the antenna mode, evidenced by the appearance of an additional antinode along the nanopost, and an increase in the field enhancement which now extends significantly into the $10-15$ range over small regions. However, this still falls short of the criterion of a field enhancement of $12-15$ over the entire surface required to be consistent with the SERS data. Moreover, insertion of the MWNT core has yet to be considered.

The second main requirement for more realistic simulation is that a MWNT core is introduced to the nanopost structures. As noted under point (3), we use the analysis of Bruna and Borini ${ }^{31}$ to yield $n_{\mathrm{MWNT}}=3.0+i 1.15$ at $\lambda=632.8 \mathrm{~nm}$. The incorporation of radial anisotropy in the MWNT is not trivial and is not taken into account at this stage; this does not deflect from the main goal here of tracking the effect of a change from a nanopost core that is metallic (Ag) to one that is (dissipative) dielectric in nature. Compared to the lumpy, solid Ag post structure of Figure 4a, that with the MWNT core (Figure 4c) exhibits slightly less field enhancement, most noticeably for the case of p-polarized light incident at $25^{\circ}$. The attenuating effect of the MWNT core inclusion is more clearly evident from a comparison of Figure $4 \mathrm{~d}$ with Figure $4 \mathrm{~b}$. The reason is some field penetration into the dissipative dielectric core, which is clearly evident in Figure 4d. In going from Figure 4 panel $c$ to $d$, the effect of greater field confinement in the higher index ambient is counteracted by dissipation in the MWNT core, yielding only a fairly marginal difference in the field enhancement. As an aside, it is pertinent to note that the $\mathrm{D}$ - and G-band signals from the MWNT core itself, due to the field penetration into the core, do not interfere with the Raman lines from CV since they are almost 4 orders of magnitude weaker.

At this juncture, there are two main points to note. First, as for the starting case of Figure $4 \mathrm{a}$, the maximum field enhancement of $6-7(+)$ in Figure $4 d$ is greater than that of the comparator cases of a smooth antenna comprising $10 \mathrm{~nm}$ of $\mathrm{Ag}$ coating on a MWNT core (4.0-4.5) or that of equivalent Ag particles on a planar $n_{\mathrm{MWNT}}$-substrate $(3.0-3.5)$, confirming the antennadriven amplification of plasmons associated with the Ag grains; as with the pure Ag nanoposts, no enhanced field moieties are sited in the antenna nodal regions. Second, there is still a gap 

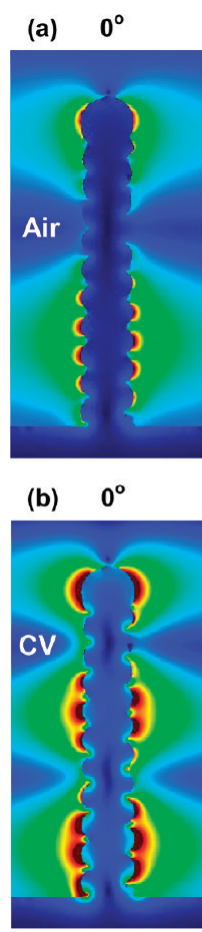

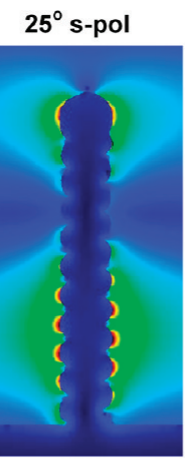

$25^{\circ}$ s-pol

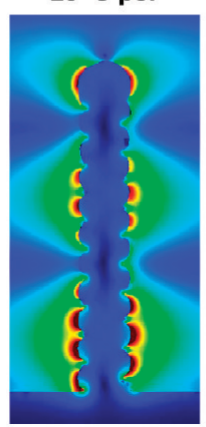

$25^{\circ} \mathrm{p}$-pol

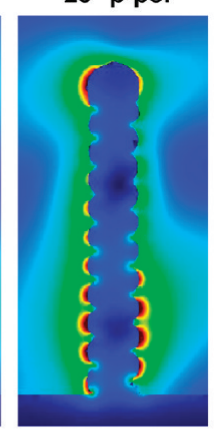

$25^{\circ}$ p-pol

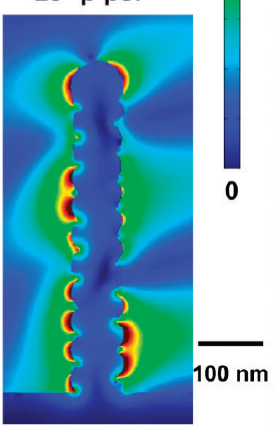

(c)

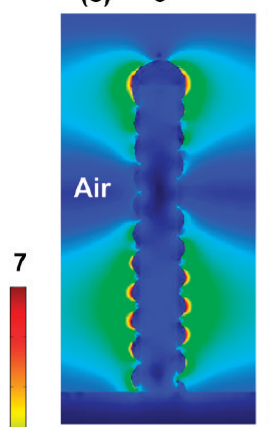

(d) $0^{\circ}$

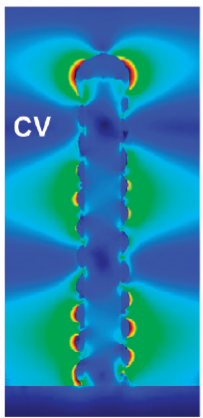

$25^{\circ}$ s-pol

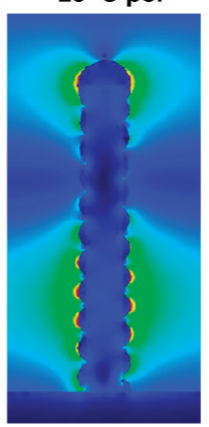

$25^{\circ}$ s-pol

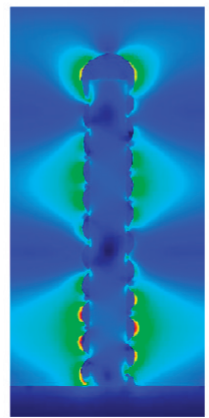

$25^{\circ}$ p-pol

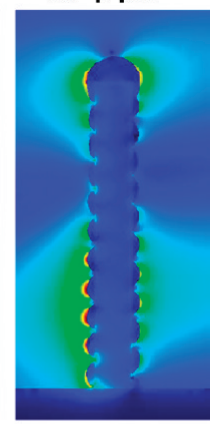

$25^{\circ} \mathrm{p}$-pol

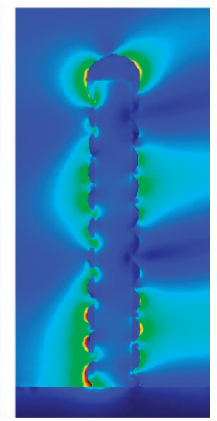

Figure 4. Electric field intensity, calculated using the SIE technique, for nanoposts with granular Ag surface structure illuminated by light of wavelength $632.8 \mathrm{~nm}$ with polarization and geometry the same as for Figure $3 . L=525 \mathrm{~nm}$ and $D=85 \mathrm{~nm}$ (to outermost edges of Ag coating) with Ag grains modeled as sections of sphere (details given in the text). (a) Granular nanopost comprised of pure Ag (i.e., no MWNT core) in ambient environment of refractive index $=1.0$ (air). (b) Granular nanopost comprised of pure Ag in ambient environment of refractive index $=1.5$, corresponding approximately to case of multilayer CV coverage. (c) Nanopost comprised of MWNT core of $60 \mathrm{~nm}$ diameter with granular Ag coating in ambient environment of refractive index = 1.0 (air). (d) Nanopost comprised of MWNT core of $60 \mathrm{~nm}$ diameter with granular Ag coating in ambient environment of refractive index $=1.5$.

between the final, simulated peak field enhancement of $6-7(+)$ of Figure $4 \mathrm{~d}$ and the average value of $12-15$ required over the entire surface to be consistent with the SERS EF in the nearsurface (monolayer) region of the nanoposts. We consider that the explanation lies with small (sub-10 nm) gaps between $\mathrm{Ag}$ particles $^{37}$ that arise from intra- and interpost structure.

Referring back to Figure 1a the dashed red lines enclose examples where the Ag coating appears as more granular on some nanoposts and thus departs from the average description used in the modeling so far. Figure 5 is presented as an example of the marked difference this can make; here the MWNT itself happens to be of slightly smaller diameter $(\sim 52 \mathrm{~nm})$ but this is of minor importance. Crucially, however, the nanopost possesses a somewhat more pronounced Ag particle structure with several nanometer gaps between the particles. Aside from more significant penetration of electric field into the MWNT core of the nanopost, it is noticeable that some of the highest field enhancements now occur in the regions between Ag grains and not just at their outer extremities. Indeed, some of the gap regions support field enhancement in the 25-30 range, offering a local SERS EF of $(6 \pm 2) \times 10^{5}$. Moreover, it appears that these hot spots are directly excited by incident light since there are occurrences in the nodal regions of the antenna field profile; this is in marked contrast with the enhanced field regions at the outer extremities of the $\mathrm{Ag}$ grains that occur only in antinodal regions of the antenna.

The second small-gap factor, also highlighted in the SEM image of Figure 1a (dashed blue circle), comprises instances of two or more leaning nanoposts coming into close proximity or touching near their tops. The effect of this feature is modeled in Figure 6 where two $85 \mathrm{~nm}$ diameter posts with $75 \mathrm{~nm}$ inside-edge-to-edge

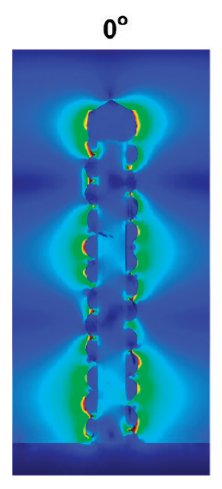

$25^{\circ}$ s-pol
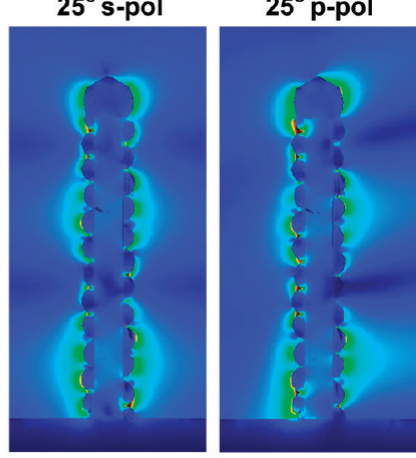

10

$100 \mathrm{~nm}$

Figure 5. Electric field intensity, calculated using the SIE technique, for nanoposts with $52 \mathrm{~nm}$ diameter MWNT core and granular Ag coating. $L=525 \mathrm{~nm}$ and $D=80 \mathrm{~nm}$ (to outermost edges of $\mathrm{Ag}$ coating) and illumination is by light of wavelength $632.8 \mathrm{~nm}$ with the polarization and geometry the same as for Figure 3. The Ag coating comprises of overlapping $\mathrm{Ag}$ particles that are almost hemispheres but with interparticle gaps of several nanometers, that is, there are small regions where the MWNT is exposed. The ambient environment is of refractive index 1.5.

spacing at the base, lean toward each other to render nanoscale gaps near their tops. With thicker Ag coating on the MWNT tops, we treat the nanoposts as pure Ag and use COMSOL to model the system with the concomitant of the posts being smooth; to give a continuous train of development from Figures $4 \mathrm{~d}$ and 5, an ambient with $n=1.5$ is used. The maximum field enhancement in 

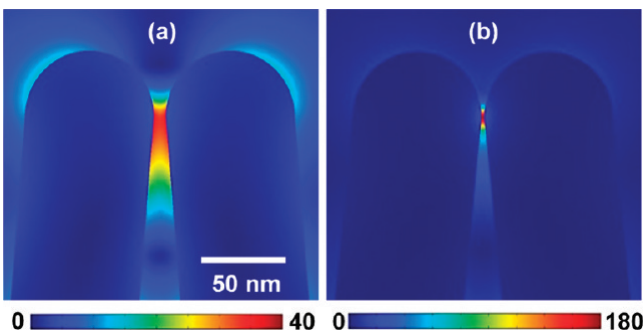

Figure 6. Plots of electric field intensity, calculated using finite element method, for two (smooth) Ag posts with $L=525 \mathrm{~nm}, D=85 \mathrm{~nm}$ with $160 \mathrm{~nm}$ center-to-center separation at the base but with minimum gap dimensions near their tops of (a) $7.5 \mathrm{~nm}$ and (b) $2.0 \mathrm{~nm}$. The ambient medium is of refractive index 1.5 and illumination is by light of wavelength $632.8 \mathrm{~nm}$ at $\theta_{i}=0^{\circ}$ with the electric field polarized in the plane of incidence.

this two-post system is $>35$ for a $7.5 \mathrm{~nm}$ gap (Figure 6a) and grows to almost 180 if the gap is reduced to $2.0 \mathrm{~nm}$ (Figure 6b). Referring back to Figure $2 b$, it is evident that the presence of neighboring posts boosts the field enhancement to some extent, but clearly when small-gap anomalies arise the field enhancement grows rapidly with the benefit to the SERS EF far outweighing the effect due to any decrease of field in the complementary, larger gaps necessarily induced elsewhere. Direct comparator cases are more difficult to specify here, but we note that (at $\lambda=$ $632.8 \mathrm{~nm}$ ) the field enhancement of Figure $6 \mathrm{~b}$ is almost the same as that offered by a dimer comprising two Ag spheres of diameter $85 \mathrm{~nm}$ and separation $2 \mathrm{~nm}$ and is significantly more than the maximum field enhancement of $\sim 80$ in $2 \mathrm{~nm}$ gaps of the nonantenna case of $85 \mathrm{~nm}$ diameter $\mathrm{Ag}$ hemispheres on a planar $\mathrm{Ag}$ surface. Thus, additional antenna-driven enhancement appears to play a role in this category of hot-spot.

Finally, it is noted that regions of extreme field enhancement between metal particles in nanometer scale proximity are understood to be the origin of single molecule SERS sensitivity, ${ }^{38}$ a feature that is especially evident in single molecule detection in tip-enhanced Raman spectroscopy. ${ }^{39}$ Also, in a study of the distribution of localized site Raman enhancements on nanostructured Ag substrates it was found that over $50 \%$ of the Raman signal originated from less than $1 \%$ of the molecules. ${ }^{9}$ Clearly, it takes only a very small percentage of hot-spot area to make a substantive difference to the average SERS EF.

Up to this point, chemical enhancement has not been considered. Here, we take this to cover both intramolecule resonance and specific molecule-metal interaction that is associated with charge transfer between the two entities. Both are relevant for $\mathrm{CV}$ under excitation at $632.8 \mathrm{~nm}(1.96 \mathrm{eV})$ since the HOMO-LUMO gap for $\mathrm{CV}$ and the molecular $\mathrm{HOMO}$ to metal Fermi-level gap occur in the range $1.9-2.0 \mathrm{eV}$, as discussed in the rigorous analysis by Canamares et al. ${ }^{40}$ In the present context, however, the reference spectra were taken from a droplet of CV solution on a Ag film, thus the chemical enhancement component is built into the reference measurement. While this means that the absolute EF is therefore underestimated in this work, the merit is that we are addressing an electromagnetic-only SERS EF in terms of only electromagnetic considerations.

In conclusion, we have modeled the electromagnetic response of a complex nanostructured substrate, comprising highly granular, MWNT-supported Ag antenna in the context of a SERS study to reveal not only (and not surprisingly) the need for hot-spots to explain the SERS EF, but a remarkable contrast in the behavior of different types of hot-spots. That contrast is between amplification, or lack of it, of the localized by the delocalized plasmonic response of the lumpy nanopost antennae, that is, between fieldenhanced regions at the outer edges of the $\mathrm{Ag}$ grains that are entirely antenna fed (no such regions occur in the antenna nodal regions) and intergrain plasmon resonances that are largely independent of the antenna response with a significant proportion occurring in antenna nodal regions. In addition, hotspots between the tops of coleaning nanoposts also seem to benefit from additional antenna enhancement. The understanding gained from these intriguing structures informs the route to forming lumpy antenna substrates for improved SERS performance. Primary factors are shorter antennae supporting a fundamental resonance (a node at the antenna base and a single antinode at the top) in the region of the excitation and scattering wavelengths, the use of a support structure that is optically less dissipative than MWNTs (pure Ag structures would clearly be superior; see Figure 4) and, more challengingly, better control over the granular $\mathrm{Ag}$ coating with a view to optimizing the nanometer-scale intergrain regions both in terms of density and uniformity.

\section{ASSOCIATED CONTENT}

S Supporting Information. The optical and geometric data of the nanopost substrates; crystal violet deposition and properties; the optical properties of MWNTs; and evaluation of the SERS enhancement factor. This material is available free of charge via the Internet at http://pubs.acs.org.

\section{AUTHOR INFORMATION}

\section{Corresponding Author}

*E-mail: P.dawson@qub.ac.uk.

\section{Present Addresses}

${ }^{\dagger}$ Present address: Depetamento de Fisica Aplicada, FCCEE, Universidad de Huelva, 21071, Spain.

\section{ACKNOWLEDGMENT}

The experimental work was supported by EC Grant STRP 505626-1 with additional financial assistance from Nanotec NI funded via Invest Northern Ireland. Support from the Swiss National Science Foundation (grant 20021-116758) is also acknowledged. P.D. is grateful for the support of a "short term scientific mission" under EC COST Action MP0803 to facilitate a period of collaborative work at EPFL, Lausanne.

\section{REFERENCES}

(1) Fleischmann, M; Hendra, P. J.; McQuillan, A. Chem. Phys. Lett. 1974, 26, 163-166.

(2) Iijima, S. Nature 1991, 354, 56-58.

(3) Goudonnet, J. P.; Inagaki, T.; Ferrell, T. L.; Warmack, R. J.; Buncick, M. C.; Arakawa, E. T. Chem. Phys. 1986, 106, 225-232.

(4) Lee, S. J.; Guan, Z. Q.; Xu, H. X.; Moskovits, M. J. Phys. Chem. C 2007, 111, 17985-17988.

(5) Jeanmaire, D. L.; Vanduyne, R. P. J. Electroanal. Chem. 1977, 84, $1-20$.

(6) Dawson, P.; Alexander, K. B.; Thompson, J. R.; Haas, J. W.; Ferrell, T. L. Phys. Rev. B 1991, 44, 6372-6381.

(7) Norrod, K. L.; Sudnik, L. M.; Rousell, D.; Rowlen, K. L. Appl. Spectrosc. 1997, 51, 994-1001.

(8) Zhang, X. Y.; Young, M. A.; Lyandres, O.; Van Duyne, R. P. J. Am. Chem. Soc. 2005, 127, 4484-4489.

(9) Fang, Y.; Seong, N. H.; Dlott, D. D. Science 2008, 321, 388-392. 
(10) Zhelyaskov, V. R.; Milne, E. T.; Hetke, J. F.; Morris, M. D. Appl. Spectrosc. 1995, 49, 1793-1795.

(11) Laurent, G.; Felidj, N.; Aubard, J.; Levi, G.; Krenn, J. R.; Hohenau, A.; Schider, G.; Leitner, A.; Aussenegg, F. R. Phys. Rev. B 2005, 71, No. 045430.

(12) Zhang, W. H.; Fischer, H.; Schmid, T.; Zenobi, R.; Martin, O. J. F. J. Phys. Chem. C 2009, 113, 14672-14675.

(13) Orendorff, C. J.; Gearheart, L.; Jana, N. R.; Murphy, C. J. Phys. Chem. Chem. Phys. 2006, 8, 165-170.

(14) Broglin, B. L.; Andreu, A.; Dhussa, N.; Heath, J. A.; Gerst, J.; Dudley, B.; Holland, D.; El-Kouedi, M. Langmuir 2007, 23, 4563-4568.

(15) Du, Y. B.; Shi, L. F.; He, T. C.; Sun, X. W.; Mo, Y. J. Appl. Surf. Sci. 2008, 255, 1901-1905.

(16) Gu, G. H.; Kim, J.; Kim, L.; Suh, J. S. J. Phys. Chem. C 2007, 111, 7906-7909.

(17) Kondo, T.; Kameshima, T.; Kawai, T. Chem. Lett. 2008, 37, 828-829.

(18) Fan, J. G.; Zhao, Y. P. Langmuir 2008, 24, 14172-14175.

(19) Balasubramanian, K.; Burghard, M. Small 2005, 1, 180-192.

(20) Ramanathan, T.; Fisher, F. T.; Ruoff, R. S.; Brinson, L. C. Chem. Mater. 2005, 17, 1290-1295.

(21) Zhao, J. J.; Park, H. K.; Han, J.; Lu, J. P. J. Phys. Chem. B 2004, $108,4227-4230$.

(22) Chhowalla, M.; Teo, K. B. K.; Ducati, C.; Rupesinghe, N. L.; Amaratunga, G. A. J.; Ferrari, A. C.; Roy, D.; Robertson, J.; Milne, W. I. J. Appl. Phys. 2001, 90, 5308-5317.

(23) Teo, K. B. K.; Chhowalla, M.; Amaratunga, G. A. J.; Milne, W. I.; Hasko, D. G.; Pirio, G.; Legagneux, P.; Wyczisk, F.; Pribat, D. Appl. Phys. Lett. 2001, 79, 1534-1536.

(24) Prodan, E.; Radloff, C.; Halas, N. J.; Nordlander, P. Science 2003, 302, 419-422.

(25) Prodan, E.; Nordlander, P. J. Chem. Phys. 2004, 120, 54445454.

(26) Gui, J. Y.; Stern, D. A.; Frank, D. G.; Lu, F.; Zapien, D. C.; Hubbard, A. T. Langmuir 1991, 7, 955-963.

(27) de los Arcos, T.; Oelhafen, P.; Mathys, D. Nanotechnology 2007, 18, No. 265706.

(28) Stagg, B. J.; Charalampopoulos, T. T. Combust. Flame 1993, 94, 381-396.

(29) Jellison, G. E.; Hunn, J. D.; Lee, H. N. Phys. Rev. B 2007, 76, No. 085125 .

(30) Blake, P.; Hill, E. W.; Neto, A. H. C.; Novoselov, K. S.; Jiang, D.; Yang, R.; Booth, T. J.; Geim, A. K. Appl. Phys. Lett. 2007, 91, No. 063124.

(31) Bruna, M.; Borini, S. Appl. Phys. Lett. 2009, 94, No. 031901.

(32) Wang, X. F.; Chen, Y. P.; Nolte, D. D. Opt. Express 2008, 16, 22105-22112.

(33) Aggarwal, R. L.; Farrar, L. W.; Diebold, E. D.; Polla, D. L. J. Raman Spectrosc. 2009, 40, 1331-1333.

(34) Le Ru, E. C.; Etchegoin, P. G.; Meyer, M. J. Chem. Phys. 2006, 125, No. 204701

(35) Kern, A. M.; Martin, O. J. F. J. Opt. Soc. Am. A 2009, 26, 732740.

(36) Yla-Oijala, P.; Taskinen, M.; Sarvas, J. Progr. Electromagn. Res. 2005, 52, 81-108.

(37) Doherty, M. D.; Murphy, A.; McPhillips, J.; Pollard, R. J.; Dawson, P. J. Phys. Chem. C 2010, 114, 19913-19919.

(38) Bosnick, K. A.; Jiang, J.; Brus, L. E. J. Phys. Chem. B 2002, 106, 8096-8099.

(39) Zhang, W. H.; Yeo, B. S.; Schmid, T.; Zenobi, R. J. Phys. Chem. C 2007, 111, 1733-1738.

(40) Canamares, M. V.; Chenal, C.; Birke, R. L.; Lombardi, J. R. J. Phys. Chem. C 2008, 112, 20295-20300. 